was undertaken in week 1 and 5 of a 5 week breathlessness programme.

Results A Total of 55 patients attended 161 session out of a potential 275 , resulting in combined total of 44 separate goals been set using the GAS Light template.

All goals were reviewed and were categorized from 3 options namely, 'a little better'; 'same' or 'worse'. 30 Goals were achieved with patients identifying them as 'a little better'. 15 goals were not achieved of those 11 remained the 'same' and 4 had got 'worse'.

23 patients did not set or complete goal setting activity due to not completing the course, declining to participate in goal setting or not returning/recording data. This is reflective of both the attendance figures and the challenges of implementing a new outcome measure in a MDT.

Conclusions Following a 5 week breathlessness programme some participants achieved and partially achieved goals important to them. Further evaluation is required.

\section{IMPROVEMENT IN PALLIATIVE CARE PRESCRIBING}

B Bonelli, D Monnery. The Clatterbridge Cancer Centre

\subsection{6/spcare-2020-PCC.202}

Background It was recognised that junior doctors in Clatterbridge Cancer Centre were unfamiliar with regional palliative care guidelines and therefore the management of common presentations of oncological and palliative care patients, resulting in delayed prescriptions, particularly out of hours.

The aim of the project was to improve knowledge of, and confidence with, the management of the most frequent palliative care issues and to improve the quality of prescribing prior to initial review by the palliative care team.

Methods Junior doctors completed a questionnaire to assess confidence in symptoms management. As baseline audit, palliative care referrals for December 2018 were reviewed retrospectively, with focus on: reasons for referral, actions carried out prior to referral and whether the interventions were appropriate and in accordance with regional guidance. These guidelines were made available on all computer desktops in March 2019, accompanied by training and communication at ward level to ensure awareness. For the post intervention measure, referrals in March 2019 were reviewed. Identical criteria were used to assess whether ready access to guidelines had improved initial patient management.

Results In December 2018, 31 out of 182 patients were referred to palliative care. Of these, 12 required review by palliative care directly for complex issues, inpatient follow-up, social/psychological support or because they were approaching end of life. Of the remaining 19 patients, $10(52.6 \%)$ had appropriate therapy initiated by junior doctors prior to palliative care review. In March 2019, 41 out of 169 patients were referred to palliative care. Of the 15 qualifying patients, 12 $(80 \%)$ were started on appropriate treatment. The percentage of referrals to palliative care for non-complex physical symptoms control dropped from $61 \%$ in December 2018 to $36 \%$ in March 2019.

Conclusions Overall, digital access to guidelines and training in their use resulted in an improvement in symptom management in inpatients.

\section{HOW ARE SPECIALIST PALLIATIVE CARE UNITS USING RANITIDINE IN THE MEDICAL MANAGEMENT OF ADULTS WITH MALIGNANT BOWEL OBSTRUCTION? A SURVEY OF UK HOSPICES}

Andrew Campbell, Fiona Rawlinson, Amy Gadoud. St Mary's Hospice (Ulverston), Cardiff University, Lancaster University

10.1136/spcare-2020-PCC.203

Background Malignant bowel obstruction (MBO) is a common presentation in palliative care and can cause challenging symptoms. It has been suggested that the H2-receptor antagonist ranitidine may have a role in the management of $\mathrm{MBO}$ as an antisecretory medication to reduce volume of gastrointestinal secretions.

Methods A questionnaire was sent via email to all UK adult hospices with inpatient units to investigate how ranitidine is currently being used for patients with MBO.

Results 60\% (99 of 165) of eligible hospices completed the questionnaire. $76 \%$ reported using hyoscine butylbromide most commonly as a first line antisecretory medication for patients with MBO.

$84 \%$ of hospices reported using ranitidine for selected patients although only $8 \%$ reported using it as a first line antisecretory agent. Hospices that use ranitidine are most commonly administering it via continuous subcutaneous infusion for patients with acid related symptoms (88\%), combined with other antisecretory medications (80\%), as a second or third line antisecretory medication (68\%) and for gastrointestinal protection (66\%). Reasons cited for not using ranitidine included a lack of perceived need, lack of evidence, non-inclusion in clinical guidelines and issues of practicality.

Responders highlighted the need for an individualised approach to prescribing in $\mathrm{MBO}$ with failure of other medications, nature of symptoms and nature of obstruction among factors influencing use of ranitidine.

Multiple hospices reported recently starting to use ranitidine or using it more frequently than they had done previously. An Australian randomised controlled trial that used ranitidine as part of a 'standard' treatment regime in $\mathrm{MBO}$ was a commonly cited instigator for change.

Conclusions Ranitidine is being used by the majority of UK hospices for selected adults with MBO despite a limited evidence base. Further research should be encouraged to evaluate the effectiveness of ranitidine and to clarify its role for patients with $\mathrm{MBO}$.

\section{COST-EFFECTIVENESS OF PULMONARY REHABILITATION: A SYSTEMATIC REVIEW}

Baldev Chahal, Deokhee Yi, Natasha Lovell, Irene J Higginson. King's College London

\subsection{6/spcare-2020-PCC.204}

Background Pulmonary rehabilitation (PR) is a complex intervention aimed at decreasing morbidity in patients with longterm lung conditions such as COPD, bronchiectasis and pulmonary fibrosis. In the NHS setting, it is an 8 week programme consisting of exercise training to reduce functional decline, and education to aid patients with self-management of their condition. Extensive evidence exists in support of the clinical effectiveness of PR but there is a paucity of studies evaluating the economics of PR. This review aimed to 